\title{
Los antibióticos resultan seguros y eficaces como tratamiento primario de la apendicitis aguda no complicada
}

Antibiotics are both effective and safe as a primary treatment for patients with uncomplicated acute appendicitis

\section{Objetivo}

Comparar la eficacia y seguridad del tratamiento antibiótico vs quirúrgico en pacientes con apendicitis aguda no complicada.

\section{Fuente de datos}

Se realizó una revisión sistemática de ensayos aleatorizados que compararon el uso de antibióticos vs apendicectomía en pacientes adultos con apendicitis aguda no complicada. Se consultaron las bases de datos Medline, Embase, librería Cochrane y Registro Cochrane de Ensayos Controlados Aleatorizados. Se evaluaron estudios publicados desde enero 1966 hasta diciembre de 2011, no li-mitados por idioma. Se incluyeron estudios con protocolos de diagnóstico y tratamiento bien definidos. Se excluyeron estudios no aleatorizados, retrospectivos, series de casos y con apendicitis complicadas (perforación local o absceso apendicular).

\section{Selección de estudios y extracción de datos}

Se incluyeron cuatro estudios con un total de 900 pacientes con sospecha de apendicitis aguda no complicada asignados al azar a dos grupos de tratamiento: antibióticos $(n=470)$ vs. apendicectomía $(n=430)$. Se analizaron como resultados primarios a las complicaciones inmediatas (infección de herida e incidencia de apendicitis perforada o peritonitis). Como resultados secundarios se evaluaron la duración de la estadía hospitalaria, los reingresos y la eficacia del tratamiento.

\section{Resultados Principales}

El diagnóstico de apendicitis se realizó de acuerdo a signos
Varadhan K y col. BMJ 2012;344:e2156 doi: 10.1136

clínicos y marcadores inflamatorios, y fue confirmado con ecografía y/o TAC en dos estudios para excluir apendicitis complicada (gas extraluminal, líquido periapendicular e intraperitoneal diseminado). La aleatorización fue realizada por computadora, estratificada por fecha de nacimiento, y la asignación fue oculta mediante sobre sellados. Ningún estudio fue ciego. La mediana de seguimiento fue de un año. Se realizó un análisis secundario excluyendo pacientes cruzados (96/202).

El meta-análisis de las complicaciones mostró una reducción del riesgo relativo del $31 \%$ en el grupo antibióticos: HR 0,69 (IC95\% 0,54 a 0,89; $I^{2}=0 \% ; p=0,004$ ). Un análisis secundario, excluyendo el estudio con mayor contaminación mostró una reducción significativa del $39 \%$ en el grupo antibióticos: HR 0,61 (IC95\% 0,40 a 0,92; $\left.\right|^{2}=0 \% ; p=0,02$ ). No se hallaron diferencias significativas en la duración de la estadía hospitalaria entre ambos grupos 0,34 (IC95\% $-0,19$ a 0,87; $12=48 \% ; p=0,20$ ). Tampoco se encontraron diferencias significativas para riesgo de apendicitis complicada 0,58 (IC95\% 0,18 a 1,90; I2=74\%; $p=0,37$ ). En el grupo antibióticos se registraron 68 de 345 (20\%) recurrencias de los síntomas. De ellos, tres fueron tratados con éxito con otro curso de antibióticos.

\section{Conclusiones}

Los antibióticos resultan seguros y eficaces como tratamiento primario de la apendicitis aguda no complicada. El tratamiento antibiótico inicial merece consideración como opción terapéutica primaria de la apendicitis aguda no complicada.

Fuente de financiamiento: Nottingham Digestive Diseases Centre NIHR Biomedical Research Unit.

\section{Comentario}

La apendicitis aguda es una de las causas más comunes de abdomen agudo. En la actualidad el tratamiento de elección para la apendicitis aguda es la apendicectomía. Sin embargo, existen complicaciones inherentes al tratamiento quirúrgico. De acuerdo a los resultados del presente estudio, en el tratamiento con antibióticos en la apendicitis aguda podría pensarse de manera similar al realizado en los pacientes con diverticulitis, donde estos juegan un papel importante en el tratamiento'. La antibioticoterapia en la apendicitis aguda también podría utilizarse como tratamiento alternativo en pacientes seleccionados o con afecciones en las que la cirugía esté contraindicada ${ }^{2,3}$. Dado que el seguimiento de los pacientes incluidos en este meta-análisis fue de un año, aún no se conoce si los pacientes tratados conservadoramente por apendicitis sin complica- ciones pueden presentar apendicitis recurrente pasado ese tiempo. Se necesitan más estudios de investigación para determinar si el tratamiento con antibióticos es más eficaz en poblaciones específicas de pacientes con un seguimiento más prolongado ${ }^{4}$.

Conclusiones del comentador

Estos resultados se deben interpretar con precaución y no es posible establecer conclusiones definitivas.

Quedaría por delimitar cuál es la pauta de tratamiento antibiótico ideal y si hay subgrupos de pacientes en los que resulta más ventajosa una u otra modalidad terapéutica.

Nadia Silvina Musarella [ Servicio de Medicina Familiar y Comunitaria. Hospital Italiano de Buenos Aires. nadia.musarella@hiba.org.ars ]

Musarella N. Los antibióticos resultan seguros y eficaces como tratamiento primario de la apendicitis aguda no complicada. Evid Act Pract Ambul Ene-Mar. 2013;(16)1:8. Comentado de: Varadhan K y col. Safety and efficacy of antibiotics compared with appendicectomy for treatment of uncomplicated acute appendicitis: meta-analysis of randomised controlled trials. BMJ 2012;344:e2156 doi: 10.1136. PMID: 22491789.

\section{Referencias}

1. Young Fadok S, Pemberton JH. Treatment of acute diverticulitis. In: UpToDate, Basow, DS (Ed), UpToDate, Waltham, MA, 2013.

2. Varadhan KK, Humes DJ, Neal KR, Lobo DN. Antibiotic therapy versus appendectomy for acute appendicitis: a meta-analysis. World J Surg 2009;34:199-209.

3. Simillis C, Symeonides P, Shorthouse AJ, Tekkis PP. A meta-analysis comparing conservative treatment versus acute appendectomy for complicated appendicitis (abscess or phlegmon). Surgery 2010;147:818-29.

4. Wilms I, de Hoog D, de Visser D, Janzing H. Apendicectomía versus tratamiento con antibióticos para la apendicitis aguda. Cochrane Database of Systematic Reviews 2011 Issue 11. Art. No.: CD008359. DOI: 10.1002/14651858.CD008359. 